ARTÍCULO DE INVESTIGACIÓN

\title{
Variación radial y axial de longitud de fibras y elementos de vaso en Nothofagus nervosa (Nothofagaceae) de la Patagonia Argentina
}

\author{
Radial and axial variation of fiber and vessel element length in \\ Nothofagus nervosa (Nothofagaceae) of Patagonia Argentina
}

\author{
Andrea A. Medina ${ }^{1^{*}}$, Natalia M. Dionisio ${ }^{1}$, Lorena N. Laffitte ${ }^{1}$, \\ Ismael R. Andía ${ }^{1}$ y Stella M. Rivera ${ }^{2}$
}

\begin{abstract}
RESUMEN
El objetivo del presente trabajo fue caracterizar las fibras y los elementos de vaso en madera de Nothofagus nervosa (Phil.) Dim. et Mil., Nothofagaceae, (raulí), determinando la variación de sus longitudes dentro del árbol, en sentido radial y axial, y evaluando la variabilidad entre árboles. Se trabajó con cuatro árboles de DAP superior a $40 \mathrm{~cm}$, del sitio Quilanlahue, Neuquén, Argentina, de los cuales se extrajeron rodajas a tres alturas distintas. Se utilizó el radio de orientación norte de cada una de ellas, realizándose preparados microscópicos y macerados cada diez años desde la médula a la corteza. Se describieron las características de fibras y elementos de vaso. La longitud de ambos tipos celulares aumentó de manera significativa en sentido radial de médula a corteza. En sentido axial la longitud de fibras aumenta, mientras que la longitud de elementos de vaso no evidencia cambios significativos. La mayor fuente de variación se presentó dentro del árbol, relacionada con las diferentes edades formativas del leño. Se observó una disminución del coeficiente de variación para la longitud de fibras y elementos de vaso a partir de los veinte años de edad del árbol. La variabilidad entre árboles no fue significativa.
\end{abstract}

PALABRAS CLAVE:

Anatomía de la madera, calidad de madera, edad del árbol, Neuquén, variabilidad de la madera.

\section{ABSTRACT}

The goal of this paper was to characterize fibers and vessel elements in the wood of Nothofagus nervosa (Phil.) Dim. et Mil., Nothofagaceae, (raulí), determining the variation of their lengths in the tree in radial and axial orientations and evaluating their variability between trees. Four trees with $\mathrm{DBH}$ (diameter at breast height) above $40 \mathrm{~cm}$ were used from Quilanlahue site, Neuquén, Argentina, from which sections were removed at three different heights. The north radius of each section was used and microscopic preparations and macerations were produced every ten years from pith to bark. The characteristics of fibers and vessel elements were described. The length of both cells was significantly greater in radial orientation from pith to bark. In axial orientation the length of the fiber is variable, while the length of vessel elements does not show significant changes. The greatest source of variation was observed in the tree related to the different ages at which wood was formed. A diminishing in the variation coefficient was observed for the length of fibers and vascular elements from twenty years of age of the tree. Variability among trees was not significant. 235, San Martín de los Andes, 8370, Provincia del Neuquén, Argentina.

2 Facultad de Ciencias Agrarias y Forestales. Universidad Nacional de La Plata. Provincia de Buenos Aires, Argentina.

* $\quad$ Autor para correspondencia. C.e.:andrepampa@yahoo.com.ar 
KEY WORDS:

Wood anatomy, wood quality, tree age, Neuquén, wood variability.

\section{INTRODUCCIÓN}

La madera es una materia prima ampliamente utilizada, aun siendo muy heterogénea y extremadamente variable. Esta variación se presenta tanto entre árboles como dentro del árbol (Larson, 1967). El tronco de un árbol maduro posee un cilindro central de madera juvenil cuyas características anatómicas, físicas y mecánicas son más variables que en la madera madura más externa. Si bien estas variaciones son moderadas en Dicotiledóneas (Dadswell, 1960; Sluder, 1972), la madera madura se presenta más estable y homogénea, con sus propiedades relativamente constantes en comparación con la madera juvenil, en la que las propiedades cambian rápidamente en dirección centrífuga.

Existen varios trabajos a nivel mundial sobre la variación de la longitud de fibras y de elementos de vaso en distintas especies y diferentes edades de Dicotiledóneas arbóreas (Denne y Whitbread, 1978; Zobel y Van Buijtenen, 1989; Wilkens, 1988; Bhat et al., 1989; Butterfield et al., 1993; Rulliaty y América, 1995; León y Espinoza de Pernía, 1999; Helinska-Raczkowska y Fabisiak, 1991; Zobel y Jett, 1995; Giménez, 2000; Moglia y López, 2001; Giménez y López, 2002; Monteoliva et al., 2006). En ellos se encuentran distintos patrones de variación, aunque el más frecuente se presenta como un incremento en la longitud de fibras y de elementos de vaso de médula a corteza y la presencia de células entre $10 \%$ y $20 \%$ más largas en la madera madura que en la madera juvenil. En sentido axial, en general, se ha encontrado una disminución de la longitud de fibras y elementos de vaso desde la base al ápice del árbol. Por otro lado, León y Espinoza de Pernía (1998) e Igartúa y Monteoliva
(2010) encontraron que la mayor fuente de variación de la morfología de las fibras se presenta dentro del árbol, relacionada con las diferentes edades formativas de la madera.

La industria y el mercado se presentan cada vez más exigentes respecto a la calidad de la madera, término estrechamente relacionado con el grado de homogeneidad de la misma. Teniendo en cuenta que la variación se acentúa en la madera que proviene de bosques nativos, los estudios focalizados en reconocer y cuantificar su variabilidad son de suma importancia. Las predicciones de calidad y usos óptimos de la madera son necesarias a la hora de planificar selecciones relacionadas con el manejo y la domesticación de las especies nativas de importancia forestal.

Si bien existen descripciones de fibras y elementos de vaso de la madera de N. nervosa (Díaz-Vaz, 1987; Rivera, 1988; Tortorelli, 2009), no se cuenta en la actualidad con información acerca de sus variaciones dentro del árbol y entre árboles ni con interpretaciones evolutivas de las mismas. Carlquist (1988) propuso el uso del índice de crecimiento intrusivo (Lf/ Lv) como indicador del grado de especialización de las maderas, ya que la divergencia entre longitud de elementos de vaso (Lv) y de elementos imperforados (Lf) se incrementa filogenéticamente.

En este marco surge la necesidad de abordar el estudio de la variabilidad de la madera del raulí (Nothofagus nervosa (Phil.) Dim. et. Mil.), importante especie forestal de área de distribución acotada en Argentina (Sabatier et al., 2011), utilizada históricamente a raíz de las buenas características estéticas y de trabajabilidad de su madera para construcciones y carpintería en general (Tortorelli, 2009).

Como fase preliminar de estos estudios en el presente trabajo se analiza la 
variación, dentro del árbol y entre árboles, de la longitud de fibras y de elementos de vaso, características que influyen significativamente en las propiedades de la madera de dicotiledóneas y por ende en el valor de su producto final (Bosman et al. 1994).

\section{OBJETIVOS}

El objetivo de este trabajo es caracterizar las células de sostén y de conducción de la madera de $N$. nervosa y determinar la variación de sus longitudes dentro del árbol, en sentido radial (edad), axial y entre árboles.

\section{METODOLOGÍA}

El estudio fue realizado en cuatro árboles de $N$. nervosa elegidos al azar, derribados en un área de bosque nativo denominado Quilanlahue, ubicado al suroeste de la provincia del Neuquén $\left(40^{\circ} 08^{\prime} 22^{\prime \prime} \mathrm{S}, 71^{\circ}\right.$ $\left.25^{\prime} 34^{\prime \prime} \mathrm{O}\right)$, Argentina, dentro del Parque Nacional Lanín (Administración de Parques Nacionales) (Fig. 1). El sitio pertenece al Distrito Fitogeográfico Caducifolio de la Provincia Subantártica (Dominio
Subantártico; Región Antártica) (Cabrera, 1976). Presenta relieve de montaña y un clima templado y húmedo de tipo mediteráneo, con precipitaciones principalmente invernales del orden de los $1800 \mathrm{~mm}$ medios acumulados anuales y temperaturas medias máximas y mínimas de $15,9^{\circ} \mathrm{C}$ y $2,8{ }^{\circ} \mathrm{C}$, respectivamente.

Los árboles elegidos (Tabla1) presentaron fuste sin defecto, estrato sociológico dominante, copa simétrica y buen estado sanitario.

Una vez derribados los ejemplares, se extrajeron rodelas completas transversales correspondientes a tres alturas, a $0,30 \mathrm{~m}$ del suelo $(\mathrm{H} 1)$, a la altura del pecho $1,30 \mathrm{~m}(\mathrm{H} 2)$ y a un tercio de la altura total $(\mathrm{H} 3)$. Las rodajas fueron pulidas hasta obtener una clara visibilidad de los anillos de crecimiento para lograr su correcto fechado. Se trabajó en cada rodela con el radio de orientación norte, del cual se realizaron macerados y preparados microscópicos permanentes mediante las normas tradicionales de la anatomía de la madera. Los preparados se obtuvieron cada diez años desde la médula a la corteza (Fig. 2).

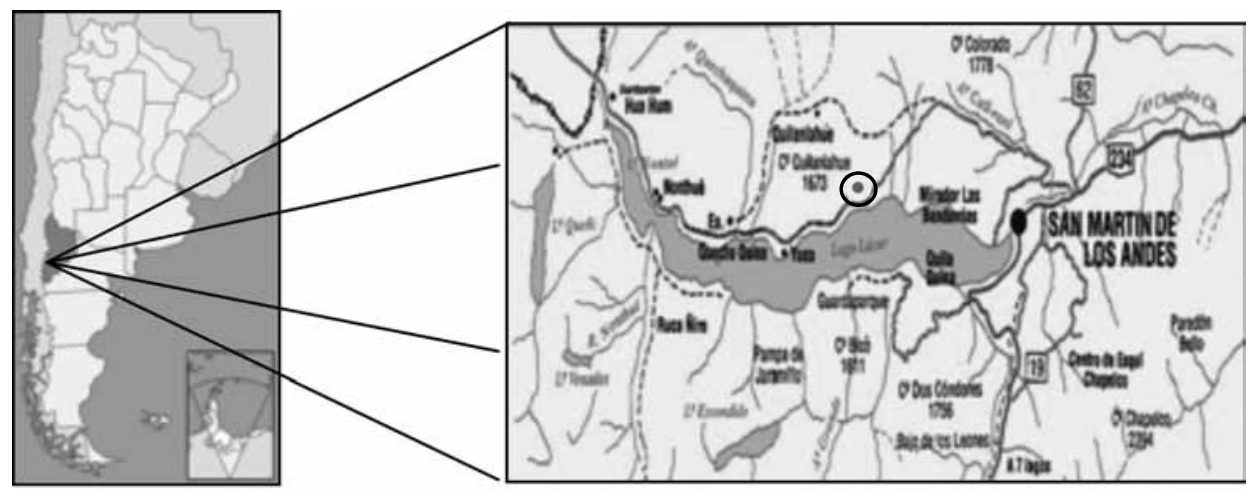

Figura 1. Mapa de la República Argentina con ubicación del sitio de procedencia de los árboles de estudio (círculo). 
Tabla 1. Datos de los árboles analizados - Magnitudes dendrométricas.

\begin{tabular}{cccc}
\hline Ejemplar & DAP $(\mathrm{cm})$ & Altura total $(m)$ & Edad (años) \\
\hline 1 & 42 & 21,8 & 60 \\
2 & 41 & 22,2 & 69 \\
3 & 42 & 23,6 & 58 \\
4 & 48 & 26,1 & 70 \\
\hline
\end{tabular}

Los macerados se realizaron mediante la técnica de Franklin (1937), a partir de los cuales se midió en microscopio óptico la longitud de 25 fibras y 25 elementos de vaso al azar por cada década y en cada altura. El material se analizó según lo establecido en la lista estándar de la Asociación Internacional de Anatomistas de la Madera para la Identificación de Maderas Latifoliadas (IAWA Commite, 1989).
Se calculó el índice de crecimiento intrusivo (longitud fibra/longitud de elemento de vaso) (Carlquist, 1992).

Se compararon los datos de longitud de fibras y de elementos de vaso mediante el análisis de varianza. La unidad experimental estuvo definida por el "macerado". La variable de respuesta quedó conformada por el promedio de las 25 mediciones
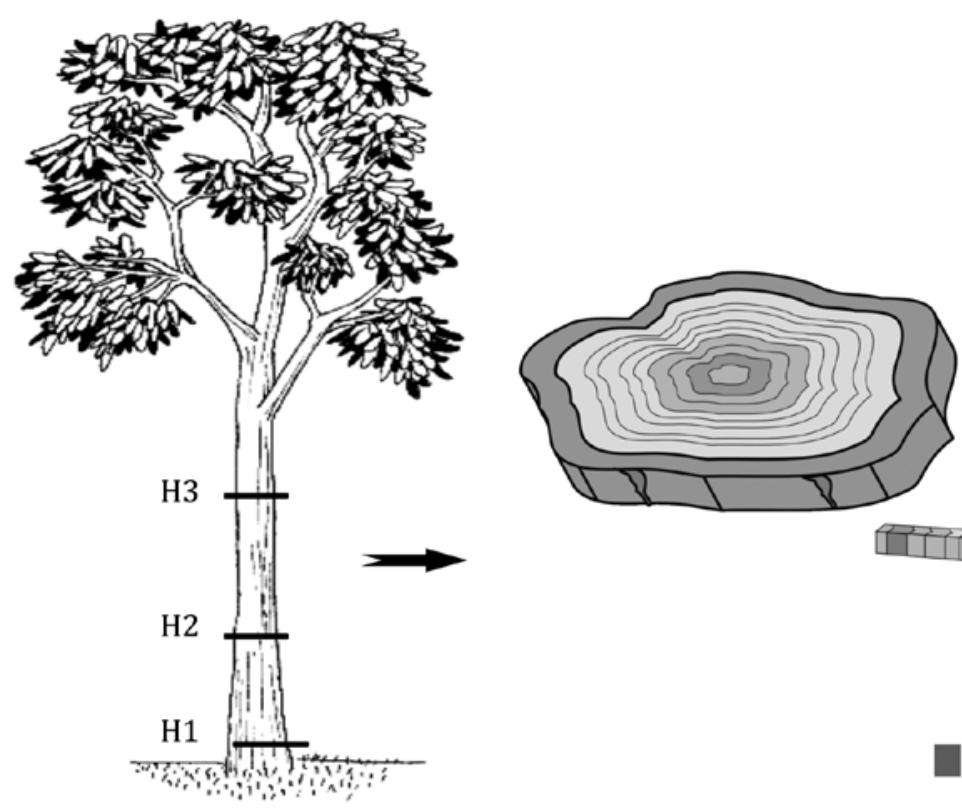

Figura 2. Esquema de toma de muestras de los árboles de estudio. De cada cubo de madera, extraído cada diez años de edad del árbol, se realizaron los preparados microscópicos y los macerados correspondientes. 
de cada macerado. Para cada variable de respuesta se ajustó un modelo lineal mixto donde las fuentes de variación estuvieron determinadas por el árbol, la altura en el fuste y la edad. El árbol fue considerado como efecto aleatorio, mientras que la altura y la edad como efectos fijos. Se verificó en cada caso el cumplimiento de los supuestos para el modelo normal y se realizaron análisis gráficos de los residuos para evaluar el ajuste. El nivel de significancia fue definido en todos los casos en $\mathrm{P}<0,05$. El modelo especificado para cada una de las variables estuvo definido por:

$$
\begin{gathered}
y_{i j k}=\mu+\alpha_{i}+\beta_{j}+\delta_{k}+\alpha \beta_{i j} \\
+\alpha \delta_{i k}+\beta \delta_{j k}+\varepsilon_{i j k} \\
i=1,2,3 j=1,2,3,4,5,6 k=1,2,3,4
\end{gathered}
$$

donde:

$y_{i j k}$ : respuesta (longitud de fibra o longitud de vaso) correspondiente al k-ésimo árbol en la i- ésima altura en la j-ésima edad.

$\mu$ : promedio general.

$\alpha_{i}$ : efecto fijo de la i-ésima altura.

$\beta_{i}$ : efecto fijo de la j-ésima edad.

$\delta_{k}$ : efecto aleatorio del k-ésimo árbol.

$\alpha \beta_{i j}$ : efecto fijo de interacción entre la i-ésima altura y la j-ésima edad.

$\alpha \delta_{i k}$ : efecto aleatorio de interacción entre la i-ésima altura y el k-ésimo árbol.

$\beta \delta_{j k}$ : efecto aleatorio de interacción entre la j-ésima edad y el k-ésimo árbol.

$\varepsilon_{i j l k}$ : error aleatorio o efecto aleatorio del k-ésimo árbol en la i-ésima altura en la j-ésima edad.

Cuando el resultado fue significativo para las diferencias de medias, se realizaron comparaciones múltiples a posteriori (prueba de Tukey) para comparar las mis- mas (Devore, 2005). Los análisis estadísticos fueron realizados con el programa Infostat (InfoStat, 2010) a través de la interfaz con $\mathrm{R}$ para modelos mixtos.

\section{RESULTADOS}

El leño de $N$. nervosa se caracteriza por presentar poca diferencia entre albura y duramen, con anillos de crecimiento levemente demarcados y de contorno regular. El anillo está definido macroscópicamente por una zona más oscura y a nivel microscópico por la compresión radial de las fibras.

La porosidad del leño es difusa, pudiéndose observar una disminución gradual del diámetro de los elementos vasculares desde el inicio hacia la finalización del crecimiento cambial anual (Fig. 3A).

\section{Fibras}

El tejido fibroso está formado principalmente por fibrotraqueidas (con puntuaciones areoladas con reborde reducido) (Fig. 3B) de diámetro medio $16,20 \mu \mathrm{m}$, diámetro medio de lumen $8,53 \mu \mathrm{m}$, espesor medio de pared $3,84 \mu \mathrm{m}$ y longitud media de $942,03 \mu \mathrm{m}$, con rango entre $640,51 \mu \mathrm{m}$ y $1.141,63 \mu \mathrm{m}$.

Se pudo observar baja presencia de fibras libriformes (con puntuaciones simples) y fibras septadas.

El análisis estadístico de la variación de la longitud de fibras indicó como significativo el efecto fijo de altura y muy significativo el efecto fijo de edad. Las interacciones dobles resultaron todas no significativas (árbol-altura; árbol-edad; altura-edad). El efecto aleatorio árbol también resultó no significativo, indicando que no hay diferencias en la variabilidad entre árboles (Tabla 2). El árbol como fuente de variación representó $12,2 \%$ de la varianza aleatoria total. 

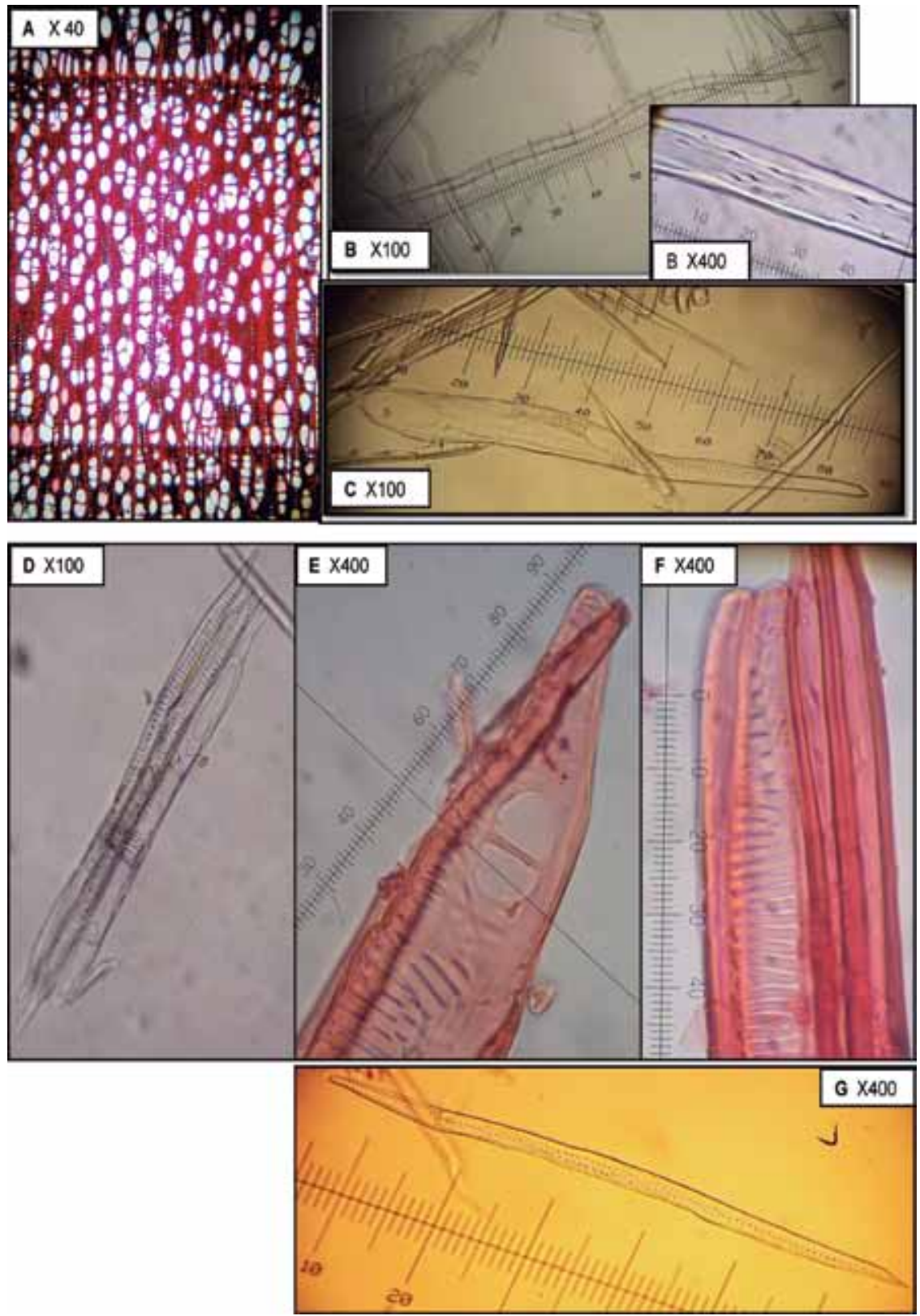

Figura 3. El leño de Nothofagus nervosa. A. Porosidad difusa; B. Fibrotraqueidas con puntuaciones areoladas con reborde reducido; C. Apéndice en elemento de vaso; D. Un elemento de vaso con placas de perforación simple y dos con placas de perforación escalariformes con 10-20 barras; E. Elemento de vaso con placa de perforación escalariforme con una barra; F. Elemento de vaso con placa de perforación escalariforme con 10-20 barras; $\mathrm{G}$. Traqueida con dos hileras de puntuaciones areoladas. 
Tabla 2. Resultados del ANOVA para la variable longitud de fibras.

\begin{tabular}{lccc}
\hline Factor de variabilidad & Grados de libertad & Prueba & $P$-valor \\
\hline & 2 & 6,95 & $0,027^{*}$ \\
Altura & 5 & 17,84 & $<0,0001^{* *}$ \\
Edad & 3 & 3,28 & $0,14 \mathrm{NS}$ \\
Árbol & 6 & 1,06 & $0,41 \mathrm{NS}$ \\
Árbol * Altura & 15 & 1,03 & $0,45 \mathrm{NS}$ \\
Árbol * Edad & 10 & 0,74 & $0,68 \mathrm{NS}$ \\
Altura * Edad & & & \\
\hline
\end{tabular}

NS: no significativo $(P>0,05),{ }^{*}$ : significativo $(0,05<P<0,01),{ }^{* *}$ : altamente significativo $(P<0,01)$.

Tabla 3. Variación radial y axial de la longitud de fibras $(\mu \mathrm{m})$. Promedios \pm desvíos estándar DE y coeficiente de variación (CV). Prueba de Tukey (significancia $\alpha=0,05$ ). Valores medios con letras distintas indican diferencias significativas, prueba de Tukey (significancia $\alpha=0,05$ ).

\begin{tabular}{lccccc}
\hline Edad & Media \pm D.E. & CV & Altura & Media \pm D.E. & CV \\
\hline 0 & $806,82 \pm 83,17$ a & 10,31 & H1 & $912,69 \pm 91,32$ a & 10,01 \\
10 & $892,42 \pm 89,03$ b & 9,98 & H2 & $983,23 \pm 107,80$ b & 10,96 \\
20 & $940,96 \pm 74,96$ bc & 7,97 & H3 & $929,66 \pm 102,14$ a & 10,99 \\
30 & $1002,59 \pm 70,44 \mathrm{c}$ & 7,03 & & & \\
40 & $1017,12 \pm 62,67 \mathrm{c}$ & 6,16 & & & \\
50 & $996,86 \pm 60,82 \mathrm{c}$ & 6,10 & & & \\
\hline
\end{tabular}

A partir de los veinte años de edad del árbol se observó una disminución del coeficiente de variación de la longitud de fibras (Tabla 3) así como la presencia de fibras $16,5 \%$ más largas.

\section{Elementos de vaso}

Los elementos de vaso poseen principalmente placas de perforación simples, terminales o laterales, horizontales $u$ oblicuos. Presentan en general apéndices (Fig. 3C), los que pueden ser de variada longitud, algunos alcanzando valores de hasta $300 \mu \mathrm{m}$. Sus puntuaciones intervasculares son opuestas, a veces escalariformes, con reborde interno de forma circular a hexagonal. La longitud media de los elementos de vaso fue de $576,14 \mu \mathrm{m}$, con un intervalo de $415,49 \mu \mathrm{m}$ a $690,82 \mu \mathrm{m}$.

En el lumen de los vasos de la madera más interna del tronco (duramen) hay abundante tílides. Se observaron elementos de vaso con placas de perforación escalariformes (Fig. 3DEF) y traqueidas con dos hileras de puntuaciones areoladas (Fig. 3G). 


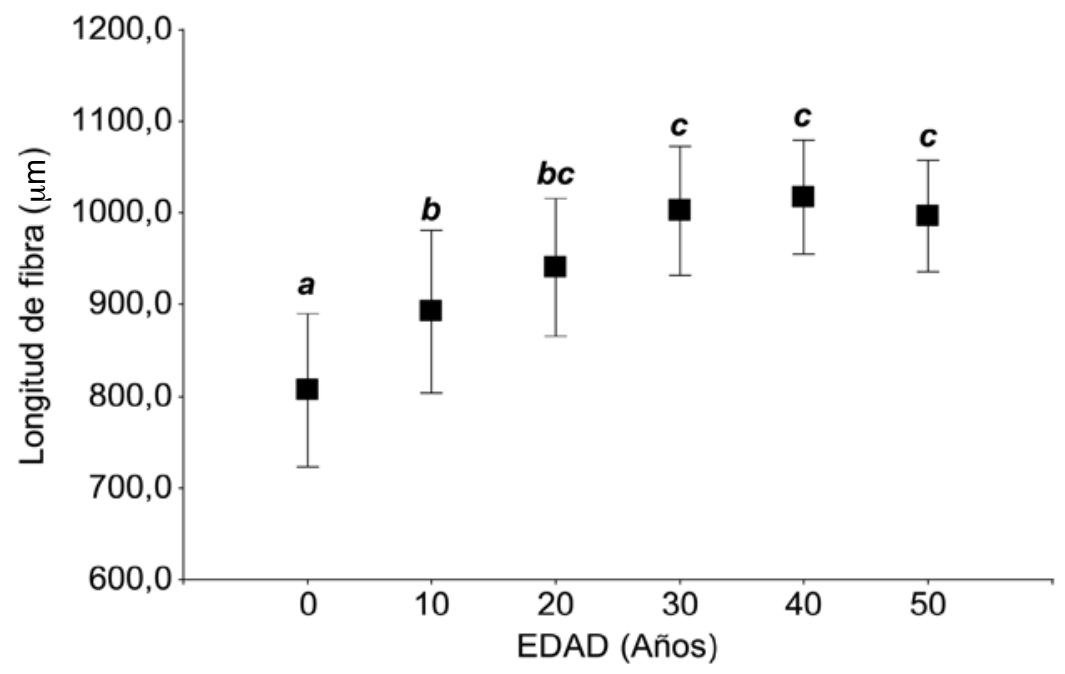

Figura 4. Variación media transversal de la longitud de fibra por edades

(Promedio + Desvío Estándar). Valores promedios con letra distinta indican diferencias significativas entre edades (Tukey, $\mathrm{P}<0,05$ ).

El análisis estadístico de la variación de la longitud de elementos de vaso indicó como significativo solamente el efecto fijo de edad. La altura del árbol no tuvo efectos significativos. El efecto aleatorio árbol también resultó no significativo indicando que no hay diferencias de variabilidad entre árboles. El árbol como fuente de variación representa menos de $1 \%$ de la varianza aleatoria total. Las interacciones dobles resultaron todas no significativas (árbolaltura, árbol-edad, altura-edad) (Tabla 4).

A partir de los veinte años de edad del árbol los elementos de vaso resultaron $18,6 \%$ más largos y el coeficiente de variación disminuyó notablemente (Tabla 5, Fig. 5). El índice de crecimiento intrusivo (Lf/Lv) arrojó un valor medio de 1,60 .

Tabla 4. Resultados del ANOVA para la variable longitud de elementos de vaso.

Factor de variabilidad Grados de libertad Prueba F P-valor

\begin{tabular}{lccc}
\hline & & & \\
Altura & 2 & 1,78 & $0,25 \mathrm{NS}$ \\
Edad & 5 & 15,60 & $<0,0001^{\text {** }}$ \\
Árbol & 3 & 0,78 & $0,54 \mathrm{NS}$ \\
Árbol * Altura & 6 & 2,19 & $0,07 \mathrm{NS}$ \\
Árbol * Edad & 15 & 1,51 & $0,16 \mathrm{NS}$ \\
Altura * Edad & 10 & 0,73 & $0,69 \mathrm{NS}$
\end{tabular}

*: significativo, ${ }^{* *}$ : no significativo, NS: no significativo 
Tabla 5. Variación radial y axial de longitud de elementos de vaso $(\mu \mathrm{m})$. Promedios \pm desvíos estándar (DE) y coeficiente de variación (CV). Valores medios con letras distintas indican diferencias significativas, prueba de Tukey (significancia $\alpha=0,05$ ).

\begin{tabular}{lccccc}
\hline Edad & Media \pm D.E. & CV & Altura & Media \pm D.E. & CV \\
\hline & & & & & \\
\hline 0 & $487,81 \pm 57,24 \mathrm{a}$ & 11,73 & $\mathrm{H} 1$ & $558,85 \pm 63,32 \mathrm{~ns}$ & 11,33 \\
10 & $538,05 \pm 51,02 \mathrm{~b}$ & 9,48 & $\mathrm{H} 2$ & $588,69 \pm 65,56 \mathrm{~ns}$ & 11,14 \\
20 & $582,66 \pm 45,19 \mathrm{bd}$ & 7,76 & $\mathrm{H} 3$ & $581,08 \pm 67,01 \mathrm{~ns}$ & 11,53 \\
30 & $611,49 \pm 27,53 \mathrm{~cd}$ & 4,50 & & & \\
40 & $632,45 \pm 35,84 \mathrm{c}$ & 5,67 & & & \\
50 & $606,93 \pm 40,93 \mathrm{~cd}$ & 6,74 & & &
\end{tabular}

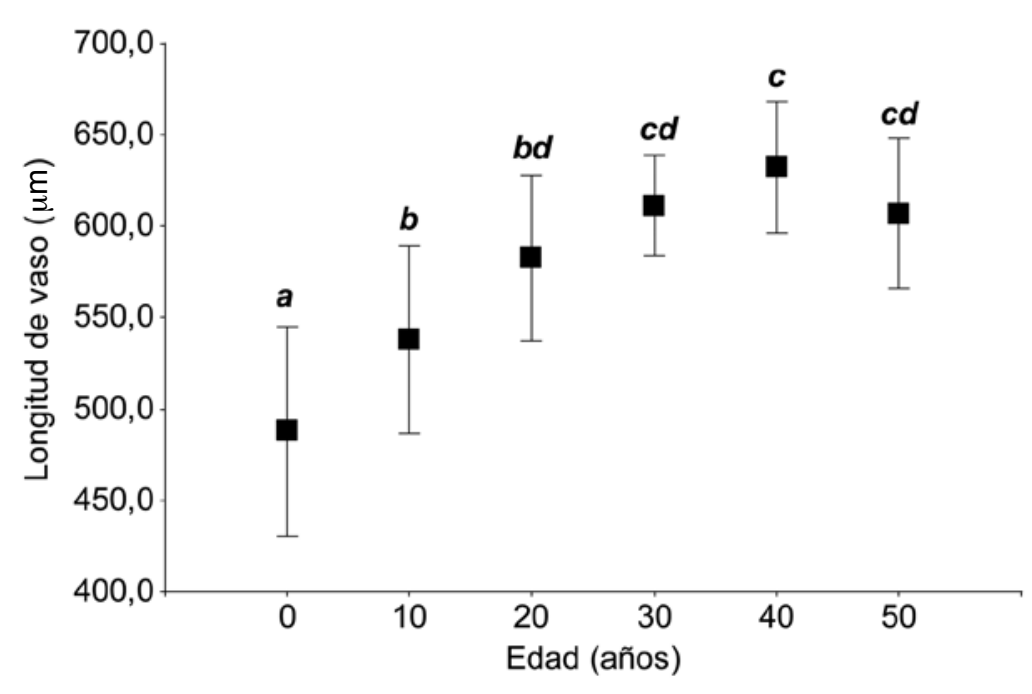

Figura 5. Variación media transversal de la longitud de elementos de vaso por edades (Promedio + Desvío Estándar). Valores promedios con letra distinta indican diferencias significativas entre edades (Tukey, $\mathrm{P}<0,05$ ).

\section{DISCUSIÓN}

Los resultados obtenidos muestran que la mayor fuente de variación de la longitud de fibras y elementos de vaso se presenta dentro del árbol, relacionada con las diferentes edades formativas del leño. Este patrón de variabilidad coincide con el des- crito por Larson (1994), en el que se señala mayor variación de las características de la madera dentro del árbol, en relación principalmente con la edad que entre árboles, aun creciendo estos en distintos sitios. León y Espinoza de Pernía (1998) e Igartúa y Monteoliva (2010) reportan similares variaciones para fibras en dicotiledó- 
neas de porosidad difusa. Claramente estos resultados están indicando un proceso de maduración del cambium vascular en $N$. nervosa. A partir de los 20 años de edad las fibras y los elementos de vaso se presentan $16,5 \%$ y $18,6 \%$ más largas, respectivamente, y muestran menores variaciones de longitud, características que señalan una mayor madurez del leño (Zobel y Van Buijtenen, 1989). HelinskaRaczkowska y Fabisiak (1991) y Moglia y López (2001) reportan la formación de madera juvenil hasta la edad de maduración sexual en Aspidosperma quebracho blanco y en especies del género Quercus, respectivamente. Las especies del género Nothofagus inician su floración y fructificación entre los 20 y los 40 años de edad (Donoso Zegers, 2006).

El patrón de incremento de la longitud de fibras, desde la médula a la corteza, encontrado en el presente trabajo es similar al descrito por Zobel y Jett (1995), Moglia y López (2001) y Giménez y López (2002) en árboles con maderas de porosidad difusa. Moglia y López (2001) describen además un incremento del $20,4 \%$ de la longitud de fibras aproximadamente a los 70 años de edad de los árboles. En este trabajo el incremento total registrado en la longitud de fibras a los cincuenta años de edad es de $24 \%$.

En géneros cercanos filogenéticamente a Nothofagus, como Quercus y Fagus, Zobel y Van Buijtenen (1989) observan patrones ascendentes continuos de longitud de fibras hasta los 100 años de edad. Por ello este trabajo deberá proseguir con ejemplares más longevos con el fin de conocer patrones en una escala temporal más amplia.

El análisis de la variación de la longitud de fibras en el eje vertical del árbol mostró valores mayores en la altura $\mathrm{H} 2$ que en las alturas $\mathrm{H} 1$ y $\mathrm{H} 3$. Este resultado difiere de los reportados por Giménez y
López (2002) y Webb (1964), en especies con leños de porosidad difusa, en los que la longitud de fibras presenta una disminución desde la base hacia el ápice del árbol. En otras especies se reportan tanto aumentos como diferencias no significativas de la longitud de las fibras con la altura del árbol (León y Espinoza de Pernía, 1999; Taylor, 1977; Lei et al., 1996).

El patrón radial de aumento de la longitud de elementos vasculares encontrado en estos árboles es similar al citado por Carlquist (1988), Larson (1994), León y Espinoza de Pernía (1998), Moglia y López (2001) y Giménez y López (2002), en especies con leños de porosidad difusa. Moglia y López (2001) describen además un aumento del $17 \%$ de la longitud de elementos de vaso a los 70 años de edad. En el presente trabajo el incremento total de la longitud de estas células a los cincuenta años de edad es de $25 \%$.

En el análisis de la variación axial de la longitud de elementos de vaso no se observan diferencias significativas, coincidiendo con los resultados reportados por Giménez y López (2002) para Schinopsis quebracho-colorado. En otras especies se reportan diferentes patrones de variación axial de los elementos de vaso (León y Espinoza de Pernía, 1999; Rulliaty y América, 1995).

La tendencia principal de la evolución del xilema señala que la divergencia entre longitud de elementos de vaso (Lv) y de elementos imperforados (Lf) se incrementa filogenéticamente, ganando estos últimos capacidad de crecimiento intrusivo a medida que maduran. Carlquist (1988) propuso el uso del índice de crecimiento intrusivo (Lf/Lv) como indicador del grado de especialización de los leños, oscilando sus valores entre 1 y 2,60 para maderas poco o altamente especializadas, respectivamente (Carlquist 1988). El bajo valor arrojado por el índice de crecimiento intru- 
sivo $(1,60)$ en este trabajo señala la presencia de una madera poco especializada en $N$. nervosa. Así lo indican también la presencia de traqueidas, de apéndices largos y placas de perforación inclinadas en los elementos de vaso y la presencia dominante de fibrotraqueidas como tejido de sostén.

\section{CONCLUSIONES}

Los resultados obtenidos justifican las siguientes conclusiones:

La mayor fuente de variación de longitud de fibras y elementos de vaso se presenta dentro del árbol, relacionada con las diferentes edades formativas del leño. La variabilidad de estos elementos entre los árboles no resulta significativa.

La longitud de fibras y elementos de vaso se incrementa significativamente en dirección a la corteza hasta la edad de 20-30 años, evidenciándose un proceso de maduración del cambium vascular. La formación de madera juvenil se extiende hasta los 20-30 años de los árboles, coincidiendo con las edades de maduración sexual citadas para la especie.

Se observa una disminución de la variación de la longitud de fibras y elementos de vaso con la edad. Asimismo, a partir de los 20 años de edad, las fibras y los elementos de vaso son $16,5 \%$ y $18,6 \%$ más largas que a edades menores. Ambas observaciones reflejan un proceso de maduración de la madera con la edad.

El valor arrojado por el índice de crecimiento intrusivo, la presencia de traqueidas, de elementos de vaso con apéndices largos y placas de perforación inclinadas y la presencia dominante de fibrotraqueidas como tejido de sostén, indica un leño poco especializado en $N$. nervosa.

\section{RECONOCIMIENTOS}

Expresamos nuestro agradecimiento a la Intendencia del Parque Nacional Lanín (APN) por facilitar acceso al sitio de estudio y al (INTA-Bariloche). Al doctor Sergio Bramardi (FCA-UNCo) por su revisión de la parte estadística y al doctor Luis Chauchard por su revisión del manuscrito. El presente trabajo fue realizado en el marco del Proyecto de Investigación S013 de la Universidad Nacional del Comahue.

\section{REFERENCIAS}

Bhat, K.M., K.V. Bhat y T.K. Dhamodaran. 1989. Fibre lenght variation in stem and branches of eleven tropical hardwoods. IAWA Bulletin 10(1):63-70.

Bosman, M.T.M., I. De Kort, M.K. Genderen y P. Baas. 1994. Radial variation in wood properties of naturally plantation grown Light Red meranti Shorea (Dipterocarpaceae). IAWA Journal 15(2):111-120.

Butterfield, R.P., R.P. Crook, R. Adams y R. Morris. 1993. Radial variation in wood specific gravity, fiber length, and vessel area for two Central American hardwoods: Hyeronima alchorneoides and Vochysia guatemalensis: natural and plantation-grown trees. IAWA Journal 14:153-161.

Cabrera, A.L. 1976. Regiones fitogeográficas de la Argentina. Enciclopedia Argentina de Agricultura y Jardinería. Tomo II. Buenos Aires.

Carlquist, S. 1988. Comparative wood anatomy. Systematic, ecological and evolutionary aspect of Dycotiledons wood. Springer. Berlin, Heidelberg, Nueva York. 460 p. 
Carlquist, S. 1992. Ecological Strategies of Xylem evolution. University of California Press.

Dadswell, H. 1960. Tree growth wood properties interrelationships. Proc.Spec. Field Inst. For. Biol. Sch. For. N. C. State Univ. Raleigh Carolina del Norte. $86 \mathrm{p}$.

Díaz-Vaz J.E. 1987. Anatomía de la madera de Nothofagus alpina (P. et. E.) Oerstedt. Bosque 8(2):143-145.

Denne, M. y V. Whitbread. 1978. Variation of fibre length within trees of Fraxinus excelsior. Canadian Journal of Forest Research 8:253-260.

Devore, J.L. 2005. Probabilidad y Estadística para ingeniería y ciencias. $6^{\mathrm{a}} \mathrm{ed}$. Thomson. México D.F. 794 p.

Donoso-Zegers, C. 2006. Las especies arbóreas de los bosques templados de Chile y Argentina. Autoecología. Marisa Cuneo Ediciones. 678 p.

Franklin, G. 1937. Permanent Preparations of Macerated Wood Fibres. Tropical woods 49:21-22.

Giménez, A.M. 2000. Gradiente radial de los elementos anatómicos del leño en Schinopsis quebracho colorado (Schelcht.) Baril et Meyer. Bosque 21(2):37-45.

Giménez, A.M. y C.R. López. 2002. Variación longitudinal de los elementos del leño en Schinopsis quebracho colorado (Schelcht.) Baril et Meyer. Madera y Bosques 8(2):27-38.

Helinska-Raczkowska, L. y E. Fabisiak. 1991. Radial variation and growth rate in the length of the axial elements of the sessile oak wood. IAWA Bulletin 12(3):257-262.
IAWA Commité. 1989. List of microscopic features for hardwoods identification. IAWA. Bulletin 10(3):219-332

Igartúa, D. y S. Monteoliva. 2010. Densidad básica, longitud de fibras y crecimiento en dos procedencias de Eucalyptus globulus en Argentina. Bosque 31(2): 50-156.

InfoStat. 2010. Di Rienzo J.A., F. Casanoves, M.G. Balzarini, L. Gonzalez, M. Tablada y C.W. Robledo. Grupo InfoStat, FCA, Universidad Nacional de Córdoba, Argentina.

Larson, P.R. 1967. Silvicultural control of the characteristics of wood used for furniture. In: Proceedings of the 4th TAPPI Forest Biology Conference, Nueva York, p:143-150.

Larson, P.R. 1994. The Vascular Cambium. Development and Structure. Springer Series in Wood Science. $720 \mathrm{p}$.

Lei, H., M. Milota y B. Gartner. 1996. Between and within tree variation in the anatomy and specific gravity in wood in Oregon white oak (Quercus garryana, Dougl.). IAWA Journal 17(4):445-461.

León, W.J. y N. Espinoza de Pernía. 1998. Variabilidad de la madera de Cordia thaisiana (Boraginaceae) en sentido transversal. Revista Forestal Venezolana 42(1):15-23.

León, W.J. y N. Espinoza de Pernía. 1999. Variabilidad de la madera de Cordia thaisiana (Boraginaceae) en sentido longitudinal. Revista Forestal Venezolana 43(1):33-41.

Moglia, J.G. y C.R. López. 2001. Variabilidad radial de algunos caracteres 
anatómicos de Aspidosperma quebracho blanco. Bosque 22(2):3-14.

Monteoliva, S., J.L. Marquina, G. Senisterra y R.M. Marlats. 2006. Variación axial y radial de la longitud de fibras en seis clones de Salix. Revista de la Facultad de Agronomía de la Plata 106(1).

Rivera, S.M. 1988. Revisión xilológica del género Nothofagus Bl. (Fagaceae) para la Argentina. Monografías de la Academia Nacional de Ciencias Exactas, Físicas y Naturales, 4:73-84. Buenos Aires, Argentina.

Rulliaty, S. y W. América. 1995. Natural variation in wood quality indicators of Indonesian Big-Leaf Mahogany (Swietenia macrophylla). http://www. metla.fi/conf./iufro95abs/d5pos9. hmt.

Sabatier, Y., M.M. Azpilicueta, P. Marchelli, M. González-Peñalba, L. Lozano, L. García, A. Martínez, L.A. Gallo, F. Umaña, D. Bran, M.J. Pastorino. 2011. Distribución natural de Nothofagus alpina y Nothofagus obliqua (Nothofagaceae) en Argentina, dos especies de primera importancia forestal de los bosques templados norpatagónicos. Boletín de la Sociedad Argentina de Botánica 46:131-138.
Sluder, E. 1972. Variation in specific gravity of yellow poplar in southern Appalachians. Wood Science 5:132-138.

Taylor, F.W. 1977. A note on the relationship between branch and stem Wood properties of selected hardwoods growing in the midsouth. Wood and Fiber 8:257-261.

Tortorelli, L.A. 2009. Maderas y bosques argentinos. Vol. I. $2^{\mathrm{a}}$ ed. Orientación Gráfica Editora. Buenos Aires. 576 p.

Webb, C. 1964. Natural variation in specific gravity, fiber length, and interlocked grain of sweetgum (Liquidambar styraciflua) in the south Atlantic Sates. Tesis doctoral School for North California State Univ. Raleigh, Carolina del Norte. 125 p.

Wilkens, J. 1988. Variation in wood anatomy within species of Eucalyptus sp. IAWA Bulletin 9:13-23.

Zobel, B.J. y J.P. Van Buijtenen. 1989. Wood variation: Its causes and control. Springer-Verlang. Berlín. 363 p.

Zobel, B. y J. Jett. 1995. Genetics of wood production. Springer-Verlag. Berlin, Heidelberg, Nueva York. 526 p. y elementos de vaso en Nothofagus nervosa (Nothofagaceae) de la Patagonia Argentina. Madera y Bosques 19(2):7-19. 